\title{
A!
}

This is an electronic reprint of the original article.

This reprint may differ from the original in pagination and typographic detail.

Räsänen, E.; Saarikoski, H.; Harju, A.; Puska, M.J.; Yu, Y.; Reimann, S.M.

\section{Giant Vortices in Rotating Electron Droplets}

Published in:

Physical Review B

DOI:

10.1103/PhysRevB.73.235324

Published: 14/06/2006

Document Version

Publisher's PDF, also known as Version of record

Please cite the original version:

Räsänen, E., Saarikoski, H., Harju, A., Puska, M. J., Yu, Y., \& Reimann, S. M. (2006). Giant Vortices in Rotating Electron Droplets. Physical Review B, 73(23), 1-5. [235324]. https://doi.org/10.1103/PhysRevB.73.235324

This material is protected by copyright and other intellectual property rights, and duplication or sale of all or part of any of the repository collections is not permitted, except that material may be duplicated by you for your research use or educational purposes in electronic or print form. You must obtain permission for any other use. Electronic or print copies may not be offered, whether for sale or otherwise to anyone who is not an authorised user. 


\title{
Giant vortices in rotating electron droplets
}

\author{
E. Räsänen, ${ }^{1,2, *}$ H. Saarikoski, ${ }^{3,2}$ Y. Yu ${ }^{4}$ A. Harju, ${ }^{2}$ M. J. Puska, ${ }^{2}$ and S. M. Reimann ${ }^{4}$ \\ ${ }^{1}$ Institut für Theoretische Physik, Freie Universität Berlin, Arnimallee 14, D-14195 Berlin, Germany \\ ${ }^{2}$ Laboratory of Physics, Helsinki University of Technology, P.O. Box 1100, FI-02015 HUT, Finland \\ ${ }^{3}$ Kavli Institute of NanoScience, Delft University of Technology, 2628 CJ Delft, The Netherlands \\ ${ }^{4}$ Mathematical Physics, Lund Institute of Technology, SE-22100 Lund, Sweden
}

(Received 25 January 2006; published 14 June 2006)

\begin{abstract}
We predict the formation of giant vortices in quasi-two-dimensional quantum dots at high magnetic fields, i.e., in rapidly rotating electron droplets. Our numerical results for quantum dots confined by a flat, anharmonic potential show ground states where vortices are accumulated in the center of the dot, thereby leading to large cores in the electron and current densities. The phenomenon is analogous to what was recently found in rotating Bose-Einstein condensates. The giant-vortex states leave measurable signatures in the ground-state energetics. The conditions for the giant-vortex formation as well as the internal structure of the vortex cores are discussed.
\end{abstract}

DOI: $10.1103 /$ PhysRevB.73.235324

PACS number(s): 73.21.La, 73.43.-f, 05.30.Fk, 03.75.Lm

\section{INTRODUCTION}

In analogy to the well-known classical phenomenon, a vortex in a quantum system can be characterized by rotational flow of particles, forming a cavity at its center. It corresponds to a node in the wave function, associated with a phase change of integer multiples of $2 \pi$ for each path enclosing it. The formation of vortices in quantum liquids has been observed, e.g., in superconductors, ${ }^{1} \mathrm{He}$ liquids, ${ }^{2}$ and more recently, rotating Bose-Einstein condensates (BECs) in atomic traps. ${ }^{3}$

Recently, electronic structure calculations of quasi-twodimensional (2D) quantum $\operatorname{dots}^{4}$ (QDs) have predicted formation of vortices in rapidly rotating electron droplets. ${ }^{5-7} \mathrm{In}$ this case an external magnetic field is used to set the system rotating. At sufficiently high fields the system tends to minimize the total energy by nucleating vortices, i.e., the quanta of the magnetic flux. This highly correlated quantum system of electrons and holelike vortex quasiparticles ${ }^{8}$ eventually shows formation of composite particles comprising an electron and a number of vortices. ${ }^{5}$ The fractional quantum Hall effect of the two-dimensional electron gas can be seen as the bulk counterpart of this phenomenon. ${ }^{9}$ The physics of rotating fermions in quantum dots shows remarkable similarities to that of rotating bosons in BECs. ${ }^{7}$ This can be seen as a consequence of the quantization of angular momentum in 2D systems.

High angular momentum of a rotating group of particles (bosons or fermions) is associated with the formation of multiple vortices, which can form as a lattice or a cluster inside the system. Another possibility is that vortices are spatially concentrated into a small area at the center of rotation. This structure is characterized by a large localized core around which the phase shift is an integer multiple of $2 \pi$. We define this core as a giant vortex. Similar structures have been observed in experiments on rapidly rotating superflow in BECs. ${ }^{10}$ It should be noted that some authors reserve the term giant vortex for multiply quantized vortices,,${ }^{11,12}$ such as those found in the Laughlin states. ${ }^{13}$ Our intention is, however, to analyze a phenomenon that is analogous to the formation of giant vortices in BECs.
In this paper we report the theoretical prediction that giant-vortex structures can be found in rotating quasi-2D fermion systems. These states are analogous to those found in BECs, and they emerge when anharmonicity (flatness) is added in the otherwise parabolic confining potential. Unlike in the bosonic case, the tendency of giant-vortex formation is limited to systems with a low number of electrons $(N<20)$. As a second difference, the cores of giant vortices in QDs do not merge completely. We show this effect using conditional wave functions to probe the internal structure of the manyelectron state. We also suggest how the formation of giant vortices could be observed in experiments.

\section{MODEL}

Our model system is a QD with electrons being restricted in a quasi-2D plane and interacting via their mutual Coulomb repulsion. The Hamiltonian is then

$$
H=\sum_{i=1}^{N}\left[\frac{\left(-i \hbar \boldsymbol{\nabla}_{i}+e \mathbf{A}\right)^{2}}{2 m^{*}}+V_{c}\left(r_{i}\right)\right]+\frac{e^{2}}{4 \pi \epsilon_{i<j}} \sum_{i j} \frac{1}{r_{i j}},
$$

where $N$ is the number of electrons, $V_{c}$ is the external confining potential on the plane, and $\mathbf{A}$ is the vector potential of the homogeneous magnetic field oriented perpendicular to the QD plane. We imitate the conditions of a real semiconductor heterostructure by using the effective material parameters for GaAs, i.e., the effective mass $m^{*}=0.067 m_{e}$ and the dielectric constant $\epsilon=12.4 \epsilon_{0}$.

We solve the many-particle Schrödinger equation for the Hamiltonian (1) using two numerical approaches, namely, the configuration interaction (CI) method which numerically diagonalizes the Hamiltonian, and the spin-densityfunctional theory (SDFT) in a real-space formulation. ${ }^{14} \mathrm{We}$ point out that our previous studies ${ }^{5,15}$ show that SDFT provides a proper description of the high-field solutions even if the total energies given by the computationally more demanding current-spin-density-functional theory are slightly closer to the quantum Monte Carlo results. ${ }^{16}$ 

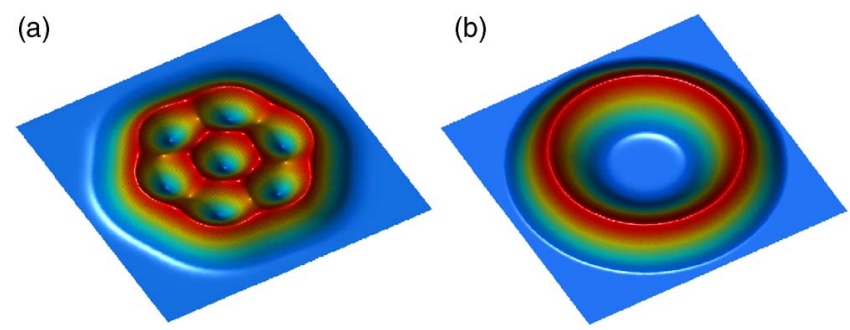

FIG. 1. (Color online) Electron densities (SDFT) of $N=10$ quantum dots containing seven flux quanta in both (a) and (b) but having different distributions of vorticity. The state in (a) is calculated using a parabolic external potential $(\hbar \omega=4 \mathrm{meV}, B=13 \mathrm{~T})$ and shows a cluster of seven vortex holes. The state in (b) is calculated in a hard-wall potential well $(R=100 \mathrm{~nm}, B=25 \mathrm{~T})$ and shows a giant core (eye) of a vortex comprising seven flux quanta close to the center of the rotation.

According to theoretical studies of rotating BECs, giantvortex structures may emerge in bosonic systems when the confining potential $V_{c}$ has a flat, nonparabolic shape. ${ }^{17-19}$ Therefore, within the CI method we apply a confinement following the notation of Jackson and co-workers: ${ }^{19}$

$$
V_{c}^{\text {quartic }}(r)=\frac{1}{2} m^{*} \omega_{0}^{2} r^{2}\left[1+\lambda\left(\frac{r}{a_{0}}\right)^{2}\right] .
$$

Here $\hbar \omega_{0}$ is the confinement strength fixed to $5 \mathrm{meV}$ unless stated otherwise, $a_{0}=\sqrt{\hbar / m^{*} \omega_{0}} \approx 15.1 \mathrm{~nm}$ is the oscillator length, and $\lambda$ is a positive dimensionless constant determining the strength of the quartic contribution, i.e., the flatness of the confinement. Within the SDFT approach we apply also a circular hard-wall potential well which can be considered as the extreme limit of the flatness of the potential. It is defined simply as

$$
V_{c}^{\mathrm{well}}(r)= \begin{cases}0, & r \leqslant R \\ \infty, & r>R\end{cases}
$$

where $R$ is the radius of the well. It should be noted that as a reasonable approximation, the confining potential in semiconductor QDs is usually assumed to be parabolic, although detailed modeling of realistic QD systems has pointed out the importance of anharmonicity in the potential. ${ }^{20}$ In addition, steep edges have been found to explain observed features in far-infrared absorption spectra. ${ }^{21}$ As we shall see below, even small deviations from the harmonicity may lead to emergence of new types of ground states.

\section{PHASE DIAGRAM}

First we visualize the fundamental difference between the high-field solutions corresponding to a vortex cluster ${ }^{5}$ and a giant vortex in a QD. Figure 1 shows the SDFT results for electron density of a vortex-cluster state (a) and a giantvortex state (b) in a ten-electron QD defined by parabolic $(\lambda=0)$ and circular hard-wall confining potentials, respectively. In the parabolic case the vorticity is spread throughout the system which gives rise to a cluster of seven holelike quasiparticles. $^{5,8}$ If the confinement is set flat, however, we find a large core in electron and current densities at the center. In this case all phase singularities are absorbed into the core region to form a single sevenfold giant vortex. The qualitative origin of the phenomenon is in the fact that increasing anharmonicity (flatness) in the external confinement leads to a relative increase in the energetic advantage of the outer orbitals compared to the inner ones. In consequence, there is rearrangement in the electron occupations such that the inner orbitals are left empty (or they have a negligible weight). Correspondingly, the vortices (holes in the electron density) are accumulated at the center.

To gain more physical insight into giant vortices, we present in the following a detailed analysis of the vortex states in a six-electron QD. In a parabolic potential the calculations have shown that the ground states in the fractional quantum Hall regime (filling factor $\nu<1$ ) occur only at certain magic values for the angular momentum $L .{ }^{22}$ For $N=6$ the $\nu=1$ state corresponds to the $L=15$ maximum-density droplet (MDD) and the subsequent magic angular momenta are $L=21,25,30,35, \ldots$. These states correspond to ground states with an increasing number of off-electron vortices forming clusters inside the electron droplet. ${ }^{5}$ When the confining potential is made flatter by including the quartic contribution of Eq. (2), new ground states emerge corresponding to giant vortices. The CI calculations show that when $\lambda>0.06$ a double-vortex core appears at angular momentum $L=27$, i.e., between the original ground states of $L=25$ and 30. A triple-vortex core emerges for $\lambda>0.16$ and it has $L$ =33. This is illustrated in Fig. 2(a) which shows the phase diagram of the CI solutions for $0<\lambda<0.2$. It should be noted that the minimum value of the quartic multiplier $\lambda$ required for giant vortices is relatively small $(\lambda \approx 0.06)$, so that the results may apply to a large class of real quantum dot devices. Figure 2(a) shows also that the giant-vortex states vanish rapidly at high fields unless $\lambda$ is considerably increased. This is due to the increasing magnetic confinement which has a parabolic form.

Figure 2(b) shows the magnetization $M=-\partial E_{\text {tot }} / \partial B$ of a QD with $\lambda=0.18$ associated with the dashed line in Fig. 2(a). The steps in $M$ correspond to changes in the many-electron ground state either due to an increase in the vortex number ${ }^{23}$ or due to a rearrangement of the vortices (and electrons) in the system. The oscillatory behavior of the magnetization might be observable in, e.g., magnetization measurements for large ensembles of QDs. Such experiments can be done using sensitive micromechanical magnetometers. ${ }^{24}$ Our data suggest, however, that the ground-state transitions associated with the rearrangement of vortices and electrons usually cause relatively small changes in the magnetization. Hence, the observation of such transitions would require a high degree of accuracy in the experiments.

\section{INTERNAL STRUCTURE OF GIANT VORTICES}

In order to visualize the internal structure of the giantvortex states, we compute the conditional wave functions ${ }^{5}$ from the numerically exact many-body ground states. Within 

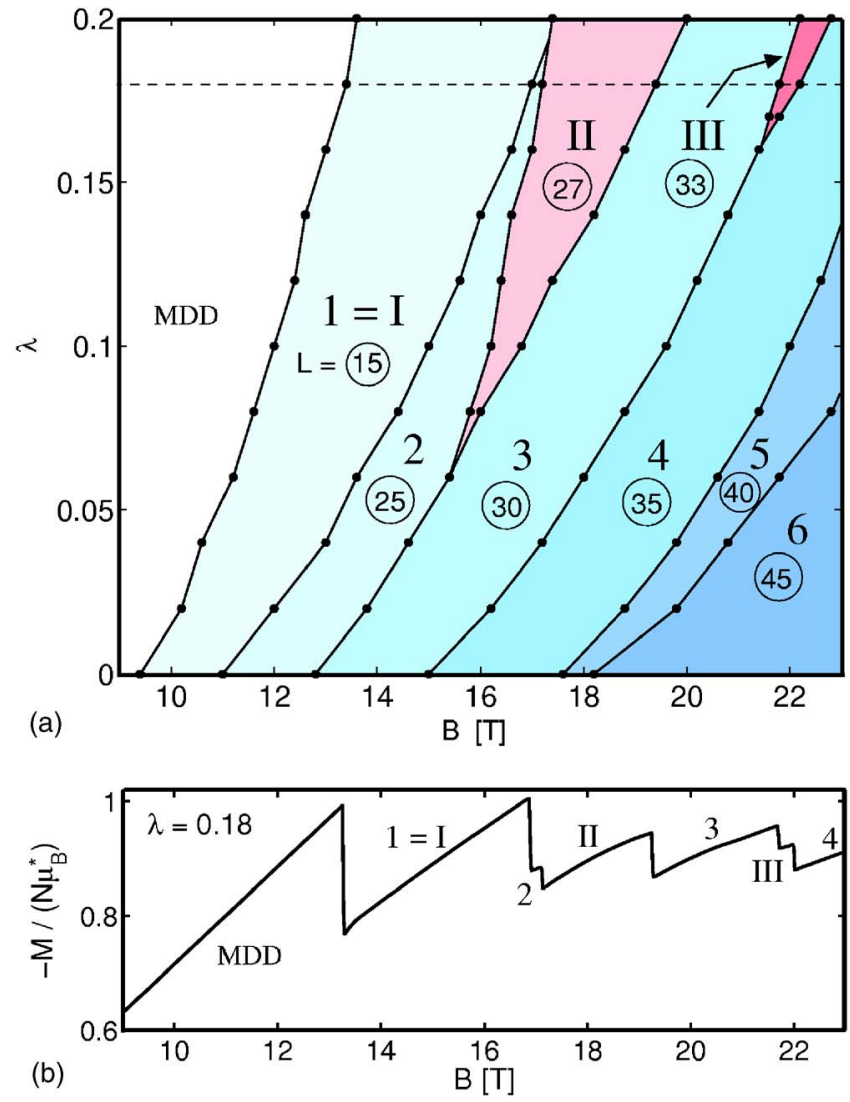

FIG. 2. (Color online) (a) Phase diagram of the vortex solutions in a six-electron quantum dot defined by a parabolic-plus-quartic confining potential [Eq. (2)]. The results have been calculated with the CI method. The roman and arabic numerals mark the number of multiple and clustered vortices in the ground states, respectively. The total angular momenta $L$ are marked by circled numbers. (b) Magnetization of a quantum dot with $\lambda=0.18$ corresponding to the dashed line in (a).

the SDFT instead, we use the auxiliary single-determinant wave function constructed from Kohn-Sham states. ${ }^{25,26}$ Figure 3 shows the total electron and current densities (left panel) and the conditional wave functions (right panel) of the double- (a) and triple- (b) vortex states at $L=27$ and 33 (CI) and an eightfold giant-vortex state (c) in a circular well with $L=63$ (SDFT). Since the system is totally polarized there is a Pauli vortex on each electron position as mandated by the exclusion principle. The exchange hole around each electron can be associated with these vortices. In addition, there are off-electron vortices close to the center of rotation. They lead to holes in the electron density with rotational current around them. The size of the vortex hole at the center of the QD increases with the number of vortices as shown in the left panel of Fig. 3.

Despite the formation of single cores in the electron and current densities, the conditional wave functions [right parts of Figs. 3(a) and 3(b)] show finite separation between the vortices at the center. The separation is due to quantum fluctuations appearing as effectively repulsive vortex-vortex interactions. This effect can also be interpreted as zero-point motion of vortices, ${ }^{26}$ which leads to nonzero electron density
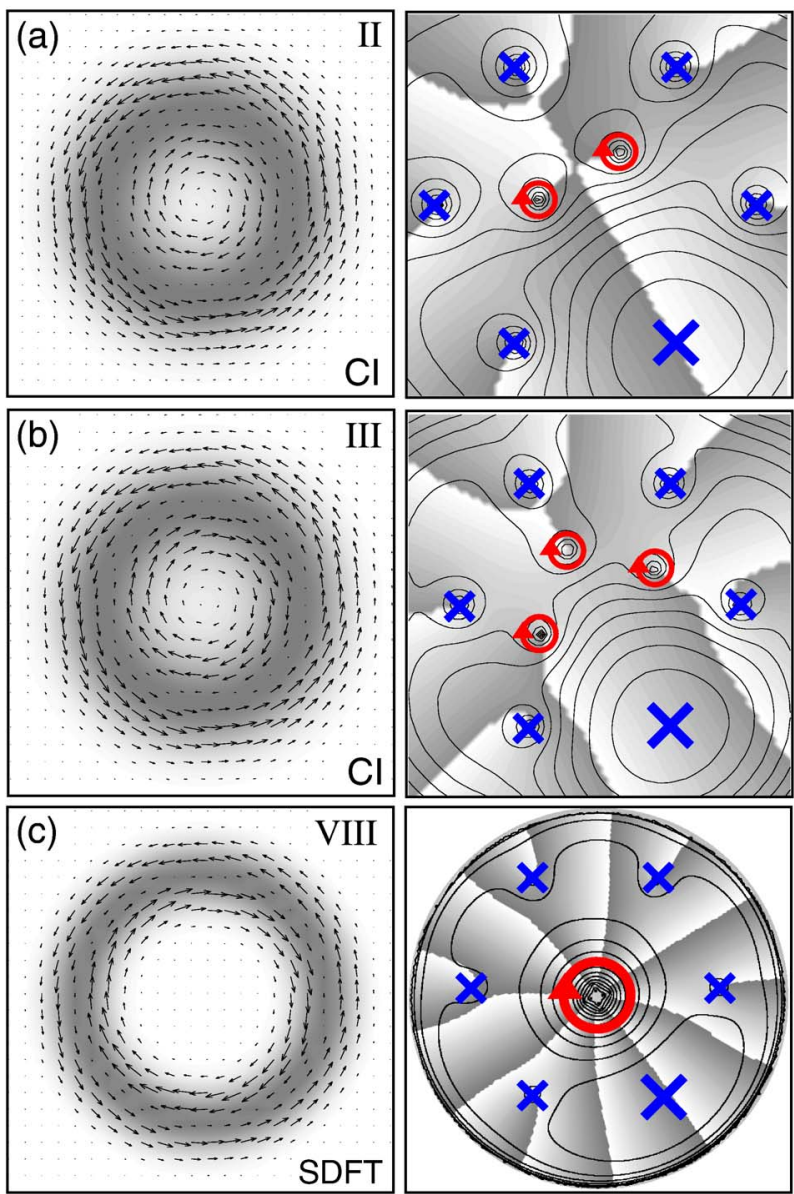

FIG. 3. (Color online) Left panel: Total electron densities (gray scale) and current densities (arrows) of double-vortex (II), triplevortex (III), and eightfold giant-vortex (VIII) states in a six-electron quantum dot. The states II and III have been calculated with the CI in a quadratic-plus-quartic potential as in Fig. 2. The state VIII has been calculated with the SDFT in a circular hard-wall well of radius $R=70 \mathrm{~nm}$. Right panel: Corresponding conditional densities (contours) and the phases (gray scale) of the conditional wave function. The phase of the SDFT result (VIII) is calculated from the singledeterminant auxiliary wave function constructed from the KohnSham states. At the lines where the shadowing changes from darkest gray to white the phase jumps from $-\pi$ to $\pi$. The crosses (blue) and circles (red) mark the most probable electron positions and vortex positions, respectively. The Pauli vortices at the electron positions are not marked by circles for clarity. The probing electron has its maximum probability downright.

at the center of the giant vortex. In the bosonic case the vortex-vortex interaction energy as a function of vortex separation has been approximated to be of logarithmic form which prevents complete merging of the vortex cores. ${ }^{18}$

In the SDFT result for the eightfold giant vortex [Fig. $3(\mathrm{c})]$, the density is zero at the center and very small up to the QD radius where the electrons are strongly localized. The vortices at the center are then packed close to each other but still show a small-scale structure. The stronger vortex localization in the SDFT can be understood from the fact that the SDFT generally can incorporate fewer correlation effects than exact many-body methods. 

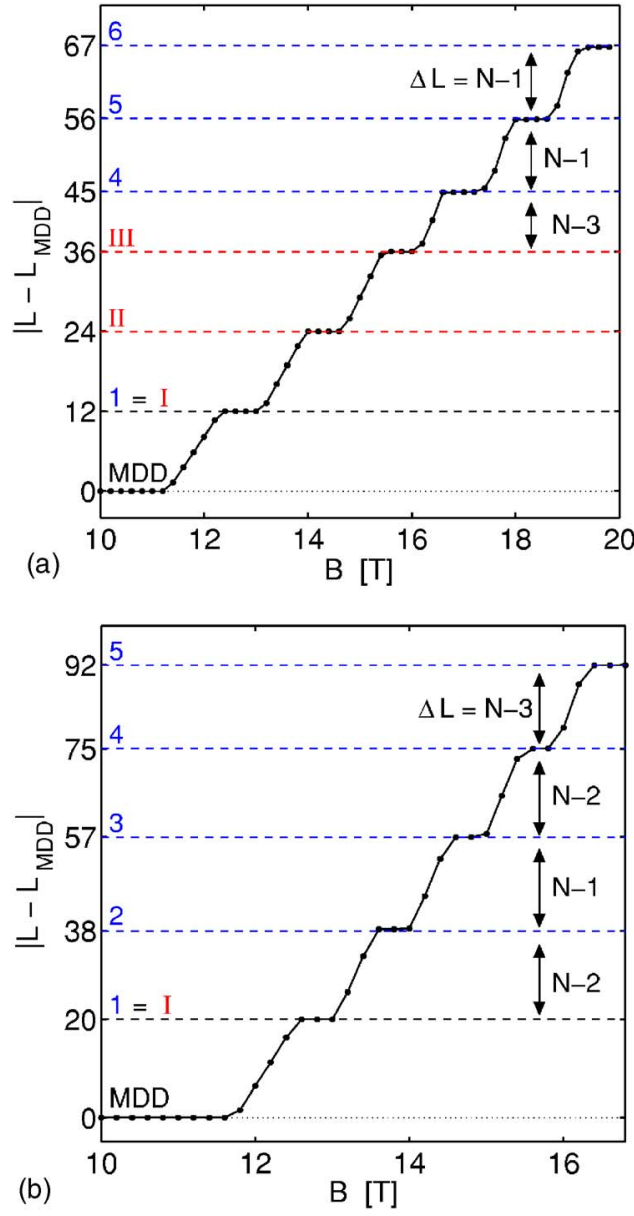

FIG. 4. (Color online) SDFT results for the total angular momenta of $N=12$ (a) and 20 (b) hard-wall quantum dots. (Red and blue) dashed lines mark the $L$ plateaus for multiple vortices (roman numerals) and vortex clusters (arabic numerals), respectively. The black dashed line corresponds to the single-vortex solution and the dotted line marks the MDD state.

Qualitatively similar internal structures have been found for giant vortices in rotating BECs. ${ }^{18}$ In that system, however, a local potential maximum at the center attracts the phase singularities into a single point in space so that a $m u l$ tiply quantized vortex is formed. ${ }^{19,27}$ This is not the case in QDs, where the confining potentials increase monotonically as a function of the radius of the system. In QDs a pinning potential, expectedly leading to perfect multiple phase quantization as in bosonic systems, could be realizable in the form of a negatively charged impurity at the center of the sample.

\section{DEPENDENCE ON THE ELECTRON NUMBER}

When $N$ is increased the maximum number of flux quanta that can be packed to the center of a giant vortex decreases rapidly. According to our SDFT calculations for hard-wall QDs [Eq. (3)] of realistic radii $(R=50, \ldots, 150 \mathrm{~nm})$, giant vortices consisting of more than ten flux quanta can be found when the electron number is increased up to $N=10$. When $N=12$, however, only three flux quanta can be packed at the center. For $N>18$ giant vortices disappear completely for realistic well sizes, and they are replaced by vortex clusters inside the electron droplet. We visualize this effect in Fig. 4, which shows the total angular momenta of the SDFT solutions for $N=12$ (a) and $N=20$ (b) hard-wall QDs as a function of the magnetic field. (Red and blue) dashed lines mark the $L$ plateaus for multiple vortices (roman numerals) and vortex clusters (arabic numerals), respectively, and the black dashed line corresponds to the single-vortex solution. The dotted line marks the MDD state. The results between the plateaus are a consequence of mixing of the consecutive exact ground states. ${ }^{5}$ In the range of the MDD and the highest multiple-vortex solution, the differences in $L$ between the plateaus are $\Delta L=N$. This regularity follows from the simple increase in the hole size discussed above. When the vortices become clustered instead, we find smaller steps in $L$ resulting from the complex rearrangements of vortex clusters in the QD.

The disappearance of giant vortices as a function of $N$ is related to the formation of shell structure in the QD, i.e., it becomes energetically favorable for the electrons to occupy the inner orbitals of the system. This behavior is in striking contrast with what is found in bosonic systems where giant vortices can be found in BECs with a very large number of particles. On the other hand, our SDFT results are consistent with the exact diagonalization results which show that even single vortices tend not to localize at the center of a parabolic QD when $N>12 .{ }^{28}$

\section{SUMMARY}

To summarize, on the grounds of our numerical analysis we predict that giant vortices emerge in quantum dots in high magnetic fields, or more generally, in rapidly rotating electron droplets. These ground states are analogous to those found in rotating Bose-Einstein condensates. In quantum dots only a slight anharmonicity (flatness) in the external confining potential is required for the formation of giant vortices. By using conditional wave functions we can analyze the internal structure of the vortex cores. Formation of giant vortices leave measurable signatures in the ground-state electron and current densities as well as in the magnetization of quantum dots. These signatures can, in principle, be detected in state-of-the-art magnetization or electron transport measurements or via direct imaging of the charge density in quantum dots. As in the bosonic case, the conditions for the emergence of giant-vortex states are determined by the interplay between the interactions and the form of the confining potential. We find that in quantum dots the giant-vortex solutions are generally limited to systems consisting of less than 20 electrons. However, our numerical analysis has been done assuming the conventional electron-electron interaction of a Coulombic form. Other types of interparticle potentials may lead to quantitatively different results, but we leave this topic for future research.

\section{ACKNOWLEDGMENTS}

We thank Matti Manninen and Georgios Kavoulakis for helpful discussions. This research has been supported by the 
Academy of Finland through its Centers of Excellence Program, the Swedish Research Council, and the Swedish Foundation for Strategic Research. E.R. acknowledges support from the NANOQUANTA NOE and from the Finnish Academy of Science and Letters, Vilho, Yrjö and Kalle Väisälä Foundation.
*Electronic address: esa@physik.fu-berlin.de

${ }^{1}$ For a review, see T. Tinkham, Introduction to Superconductivity (McGraw-Hill, New York, 1996).

${ }^{2}$ V. M. H. Ruutu, V. B. Eltsov, A. J. Gill, T. W. B. Kibble, M. Krusius, Y. G. Makhlin, B. Plaçais, G. E. Volovik, and W. Xu, Nature (London) 382, 334 (1996).

${ }^{3}$ D. A. Butts and D. S. Rokhsar, Nature (London) 397, 327 (1999); M. R. Matthews, B. P. Anderson, P. C. Haljan, D. S. Hall, C. E. Wieman, and E. A. Cornell, Phys. Rev. Lett. 83, 2498 (1999); K. W. Madison, F. Chevy, W. Wohlleben, and J. Dalibard, ibid. 84, 806 (2000); J. R. Abo-Shaer, C. Raman, J. M. Vogels, and W. Ketterle, Science 292, 476 (2001).

${ }^{4}$ For a review, see, e.g., L. P. Kouwenhoven, D. G. Austing, and S. Tarucha, Rep. Prog. Phys. 64, 701 (2001); S. M. Reimann and M. Manninen, Rev. Mod. Phys. 74, 1283 (2002).

${ }^{5}$ H. Saarikoski, A. Harju, M. J. Puska, and R. M. Nieminen, Phys. Rev. Lett. 93, 116802 (2004).

${ }^{6}$ M. B. Tavernier, E. Anisimovas, and F. M. Peeters, Phys. Rev. B 70, 155321 (2004).

${ }^{7}$ M. Toreblad, M. Borgh, M. Koskinen, M. Manninen, and S. M. Reimann, Phys. Rev. Lett. 93, 090407 (2004).

${ }^{8}$ M. Manninen, S. M. Reimann, M. Koskinen, Y. Yu, and M. Toreblad, Phys. Rev. Lett. 94, 106405 (2005).

${ }^{9}$ T. Chakraborty and P. Pietiläinen, The Quantum Hall Effects: Fractional and Integral (Springer, Berlin, 1995).

${ }^{10}$ A. E. Leanhardt, A. Görlitz, A. P. Chikkatur, D. Kielpinski, Y. Shin, D. E. Pritchard, and W. Ketterle, Phys. Rev. Lett. 89, 190403 (2002); P. Engels, I. Coddington, P. C. Haljan, V. Schweikhard, and E. A. Cornell, ibid. 90, 170405 (2003).

${ }^{11}$ A. Kanda, B. J. Baelus, F. M. Peeters, K. Kadowaki, and Y.
Ootuka, Phys. Rev. Lett. 93, 257002 (2004).

${ }^{12}$ T. Stopa, B. Szafran, M. B. Tavernier, and F. M. Peeters, Phys. Rev. B 73, 075315 (2006).

${ }^{13}$ R. B. Laughlin, Phys. Rev. B 27, 3383 (1983).

${ }^{14}$ For a review of the implementation of our SDFT method, see T. Torsti et al., Phys. Status Solidi B 243, 1016 (2006).

${ }^{15}$ E. Räsänen, A. Harju, M. J. Puska, and R. M. Nieminen, Phys. Rev. B 69, 165309 (2004).

${ }^{16}$ H. Saarikoski, E. Räsänen, S. Siljamäki, A. Harju, M. J. Puska, and R. M. Nieminen, Phys. Rev. B 67, 205327 (2003).

${ }^{17}$ E. Lundh, Phys. Rev. A 65, 043604 (2002).

${ }^{18}$ K. Kasamatsu, M. Tsubota, and M. Ueda, Phys. Rev. A 66, 053606 (2002).

${ }^{19}$ A. D. Jackson, G. M. Kavoulakis, and E. Lundh, Phys. Rev. A 69, 053619 (2004); A. D. Jackson and G. M. Kavoulakis, ibid. 70, 023601 (2004).

${ }^{20}$ P. Matagne and J.-P. Leburton, Phys. Rev. B 65, 235323 (2002).

${ }^{21}$ See C. A. Ullrich and G. Vignale, Phys. Rev. B 61, 2729 (2000), and references therein.

${ }^{22}$ P. A. Maksym and T. Chakraborty, Phys. Rev. Lett. 65, 108 (1992).

${ }^{23}$ H. Saarikoski and A. Harju, Phys. Rev. Lett. 94, 246803 (2005).

${ }^{24}$ M. P. Schwarz, D. Grundler, C. Heyn, D. Heitmann, D. Reuter, and A. Wieck, Phys. Rev. B 68, 245315 (2003).

${ }^{25}$ H. Saarikoski, A. Harju, M. J. Puska, and R. M. Nieminen, Physica E (Amsterdam) 26, 317 (2005).

${ }^{26}$ H. Saarikoski, S. M. Reimann, E. Räsänen, A. Harju, and M. J. Puska, Phys. Rev. B 71, 035421 (2005).

${ }^{27}$ U. R. Fischer and G. Baym, Phys. Rev. Lett. 90, 140402 (2003).

${ }^{28}$ A. Harju, J. Low Temp. Phys. 140, 181 (2005). 